Running head: Musculoskeletal pain in adolescents and young adults

Baseline musculoskeletal pain and impaired sleep related to school pressure influence the development of musculoskeletal pain in $\mathrm{N}=107$ adolescents in a five-year longitudinal study 


\begin{abstract}
(150 to max. 250 words)
Purpose: This longitudinal study followed 10- to 13-year-old adolescents for five years to investigate the effects of juvenile musculoskeletal (MSK) pain and psychosocial risk factors on future pain. We further predicted that increased MSK pain at follow-up would be positively related to current school pressure at follow-up and negatively related to current sleep quality. Sleep quality was tested as a potential mediator of the link between school pressure and MSK pain at follow-up after controlling for baseline MSK pain.

Methods: The baseline sample comprised 189 adolescents and five-year follow-up resulted in 107 15- to 18-year-old adolescents who had completed mandatory education. Adolescents responded to an online questionnaire about psychosocial stressors, MSK pain, school achievement, and leisure activities. A longitudinal hierarchic linear regression including all significant baseline predictors was run to assess their impact on MSK pain five years later. Mediation analysis was used to investigate sleep quality as a potential mediator of the relationship between school pressure and MSK pain at follow-up.
\end{abstract}

Results: Baseline MSK pain predicted MSK pain over a time lag of five years $(\beta=.26, p=.02)$. The relationship between follow-up school pressure and current MSK pain was mediated by sleep quality at follow-up $\left(\mathrm{B}=.17, \mathrm{SE}_{\mathrm{B}}=.07, \mathrm{CI}-95=.06\right.$ to .34$)$ when baseline MSK pain was controlled.

Conclusions: Juvenile MSK pain predicts MSK pain in adolescence. A psychosocial mediation model including school pressure and sleep impairments has the potential to explain MSK pain mechanisms in adolescents.

\title{
4-6 Keywords:
}

Musculoskeletal pain, longitudinal analysis, adolescents, psychosocial risk factors, sleep quality, school characteristics 


\section{Baseline musculoskeletal pain and impaired sleep related to school pressure influence the development of musculoskeletal pain in $\mathrm{N}=\mathbf{1 0 7}$ adolescents in a five-year longitudinal study"}

Non-specific musculoskeletal (MSK) pain is considered common in adolescents, with estimated prevalence ranging from $7 \%$ to as high as $72 \%$, with a mean of $40 \%$ [1] and previous pain episodes triggering further episodes [2]. The prevalence of MSK pain approaches adult levels by the end of adolescence [3], increasing at 11 to 12 years, i.e. around the onset of puberty [1]. Adolescent girls generally report higher prevalence and incidence than boys [2, 4-6]. Furthermore, various environmental and psychosocial risk factors, including psychological distress, seem to be more strongly related to MSK pain in children and adolescents than genetic factors $[2,7]$. However, the evidence remains unclear on factors such as increased screen viewing time [1]. Methodological limitations, such as the lack of longitudinal data and the heterogeneity of outcome measures, account for gaps in knowledge about juvenile risk factors for MSK pain [1]. Longitudinal studies are therefore warranted.

There has been little scientific research on associations between MSK pain and specific psychosocial factors such as school performance, school grades and pressure to do well at school. It has long been hypothesised that there is a link between school performance and MSK pain in children or adolescents [8], but the possibility was barely mentioned in two systematic reviews [2, 4] and supporting evidence has only recently begun to emerge, for example in a cross-sectional study [9] examining 189 fifthgraders aged between 10 and 13 years. That study [9] pointed out that MSK pain was related to psychosocial strain, school-type recommendation and unsatisfactory grades in mathematics. Furthermore, two recent, cross-sectional Chinese studies investigated two large samples of Chinese middle and high school students and demonstrated that MSK pain was associated with school pressure, homework overloading and perceived stress [10-11].

Development of MSK pain in adolescents has also been associated with perceived stress [12-13]. More precisely, school-related stress such as academic stress, difficulties with peers at school, or schoolwork pressure was shown to be related to subjective health complaints that were defined as somatic complaints, for example irritability, nervousness/being afraid, headache, abdominal pain, backache and depressed mood [14-15]. Such complaints often contribute to short-term - as opposed to chronic - sleep problems, lack of sleep and poor sleep quality, which are all known to lower pain thresholds [16]. Hence, a 
Musculoskeletal pain in adolescents and young adults 4

mediation of the link between school pressure and MSK pain by short-term sleep problems is to be expected.

This study represents a continuation of the study of Erne and Elfering [9] in the form of a five-year follow-up. The longitudinal data allowed us to test the extent to which juvenile MSK predicted MSK. Additionally, we aimed to investigate the relationships between developments of MSK pain, school pressure, and sleep quality in adolescents.

\section{Method}

\section{Design and Hypothesis}

This observational study had two measurement points: baseline and a five-year follow-up. We hypothesised that significant psychosocial predictors in the baseline study would have a longitudinal effect on the development of MSK pain over the five-year study period. We also hypothesised that the relationship between current academic pressure and the development of MSK pain would be mediated by current short-term subjective sleep quality.

\section{Sample}

The baseline study was conducted in 2008 and examined 11 school classes of 189 fifth-graders aged between 10 and 13 years [9]. Subjective statements were collected with a paper pencil questionnaire and teacher reports on school grades and recommended school type were also collected [9]. In preparation for the follow up assessment primary schools were asked to provide addresses of former pupils in accordance with Swiss data protection law. Swiss law allows schools to refuse to supply such data without giving a reason; one of the seven schools that had participated in the baseline assessment [9] did this $(N=$ 15). Of the remaining $N=174$ addresses, $N=50$ were invalid or no longer accurate, so that we were only able to contact $N=124$ participants. Out of these, $\mathrm{N}=17$ refused to take part which left us with a follow-up sample of $N=107$ (86.3\% retention rate) 15 - to 18 -year-old adolescents $(M=16.3$ years $\pm 0.7,66 \%$ females) who consented by telephone and provided e-mail address in early 2013 (Figure 1). After participants had consented to participate they were sent a link to an online questionnaire via email. The online questionnaire was identical, yet slightly supplemented, to the paper pencil questionnaire that was used in the baseline assessment. Participants who did not follow the link and complete the questionnaire within 24 hours were contacted by email again and those who had still not completed the questionnaire 
Musculoskeletal pain in adolescents and young adults 5

after 48 hours were contacted by telephone. All participants were entered into a draw for two concert tickets when they submitted their data.

\section{Materials}

The following significant baseline predictor variables [9] were included as potential prospective risk factors in longitudinal analysis.

Pain assessment. At follow-up total MSK pain was assessed using blank-faced, genderless pain mannequins [17] on which participants were asked to mark all current pain sites and all pain sites in which they had experienced pain for at least a day during the last four weeks by ticking a labelled radio button. Scores from all pain sites were summed. The same pain variable was assessed during baseline data assessment [9]. Furthermore, pupils were asked about parental back pain at baseline [9].

Anthropomorphic factors. Variables assessed were age, gender, and body mass index (BMI).

School variables. Satchel weight was measured with a digital scale to an accuracy of $100 \mathrm{~g}$. Recommended school type was derived from the fact that, at the end of primary school (International Standard Classification of Education: ISCED level 1; 12-13 years) pupils are recommended to enrol in one of three different performance-based secondary schools (ISCED level 2): basic, expanded lower-secondary or expanded higher-secondary, based on their grades and performance in achievement tests.

\section{Daily media consumption.}

Participants reported the total daily media consumption (six point scale ranging from 0 : none to $5:>3$ hrs/day).

Psychosocial strain. Psychosocial problems were assessed with the German version of the Strengths and Difficulties Questionnaire for 11- to 16-year-olds (SDQ; [18]), with 20 items using a three-point scale ( $0=$ no, $1=$ sometimes, 2 =yes). We calculated mean scores for each dimension. A reliability test showed that Cronbach's alpha was satisfactory $(\alpha=.74)$.

The mediation analysis used follow-up data on two variables, school pressure and sleep quality, with MSK pain as the outcome variable.

School pressure at follow-up. Participants evaluated the amount of pressure they felt to achieve good school grades (Five-point Likert scale ranging from $1=$ no pressure to $5=$ very intense pressure [9]). 
Musculoskeletal pain in adolescents and young adults 6

Sleep quality at follow-up. Participants reported subjective short-term sleep quality using a five-point Likert scale ranging from $1=$ very poor to $5=$ very high [16].

\section{Data Analysis}

A longitudinal hierarchic linear regression with MSK pain at follow-up as the outcome variable was conducted, using SPSS Version 25 (IBM SPSS Inc., Armonk, NY). First, total baseline MSK pain was entered to model the influence of previous MSK pain episodes. Then, in a second and third step, in order to replicate the baseline analysis, all significant baseline predictors were entered [9]. All $p$ values in the hierarchical regression analysis were two-tailed, with $\alpha$ set to $5 \%$.

Also, a mediation analysis was carried out to investigate the associations between school pressure, sleep quality and total MSK pain at follow-up using Hayes' [19] bootstrap test with 5,000 re-samples for estimation of indirect effects (PROCESS macro for SPSS, model 4) using 95\% confidence intervals (CIs) to assess mediation effects. If the upper and lower bounds of the $95 \%$ bias-corrected CI do not contain zero an indirect effect is considered significant. The bootstrapping method recommended by Preacher and Hayes [20] is acknowledged as the most powerful and effective method in small samples and is also least vulnerable to Type I errors.

\section{Results}

A baseline comparison of completers and non-completers shows whether a potential bias from dropouts is likely. The age ( $M=16.3$ years, $S D=0.7$ years) and gender distribution ( $66.4 \%$ female) of completers at baseline were similar to those for participants lost to follow-up, so were all other predictors included in the longitudinal hierarchic linear regression analysis but one. Completers were significantly recommended to a higher school grade than non-completers $(t(187)=2.58, p<.05)$. At follow-up the period prevalence of MSK pain over the last four weeks was $73.8 \%$, compared with $61.9 \%$ at baseline (Table 1). Of the 61 participants (65.3\%) who had reported MSK pain at baseline, 45 also experienced MSK pain at follow-up. Thirty of the 46 pain-free participants at baseline experienced MSK pain at follow-up, whereas 16 remained pain-free $\left(X^{2}(1, N=107)=0.92, \mathrm{p}=.34\right)$.

It is notable that there was a slight increase in reported spinal pain, and a markedly increase of daily media consumption (Table 1). 
Musculoskeletal pain in adolescents and young adults 7

There were high correlations between sleep quality and MSK pain at follow-up $(r=-.48, p<$ $.001)$. Recommended school type was also negative correlated with psychosocial strain $(r=-.46, p<.001)$.

Only one of the baseline predictors investigated actually predicted MSK pain at follow-up, namely baseline MSK pain $(\beta=.26, p=.02)$. However, there is a notable tendency regarding female gender predicting MSK pain over the time span of five years (Table 3).

The proposed mediation model was confirmed by empirical data. The association between current school pressure and total MSK pain at follow-up was mediated by sleep quality, even when all covariates were included in the equation. Figure 2 shows the model paths and estimates of unstandardized path coefficients. The indirect path from school pressure to MSK pain at follow-up via sleep quality was significant $\left(\mathrm{B}=.17, \mathrm{SE}_{\mathrm{B}}=.07, \mathrm{CI} 95 \%: .06-.34\right)$.

\section{Discussion}

The results of the longitudinal analysis confirmed baseline MSK pain as a risk factor for the development of MSK pain over the next five years [2-3], but none of the baseline psychosocial predictors foretold total MSK pain at follow-up. The mediation analysis demonstrated that baseline school pressure and poor sleep quality were risk factors for MSK [9]. In line with expectation, the relationship between school pressure, operationalised as perceived pressure to obtain good grades, and total MSK pain was mediated by current sleep quality, even after controlling for variance in baseline MSK pain. Mediation suggests that current psychosocial stress and sleep impairments contribute to current MSK pain beyond baseline levels of MSK pain.

Consistent with previous research we found that in adolescents a history of MSK pain is one of the main predictors of future MSK pain episodes [2-3]. For example, almost $74 \%$ of those who were suffering of MSK pain at baseline also reported MSK pain at the five-year follow-up and an additional 30 participants who had been MSK pain-free at baseline also reported MSK-pain at follow-up, with only 16 remaining pain-free. Thus, our data demonstrate the importance of previous MSK pain episodes as predictors of new episodes, and the rapid rise in prevalence of MSK pain from childhood to adolescence [3]. With regard to female gender as weak predictor of MSK pain, the study showed some tendency in this direction and results may have been more conclusive in a larger sample [6]. Further investigations need to take into account possible accompanying psychosocial, gender-specific factors. 
Musculoskeletal pain in adolescents and young adults 8

It is notable that MSK pain at follow-up was not associated with any of the baseline psychosocial predictor variables for MSK pain. In contrary, the SDQ demonstrates slightly, but insignificantly lower results at follow-up. This might be due to the fact that baseline assessment took place during a very demanding and stressful period of time for the pupils with the secondary school entry test ahead [9]. Also, by the age of 15.5 to 18 years, for most adolescents puberty is slowly fading out and the course has been set for the first career choice. Being in the midst of a stable continuing education process, the perceived stress level might be somewhat lower. Thus, psychosocial stressors in adolescents potentially elicit stress reactions that can explain some variation in not yet chronic or genetically-bound MSK pain. Thinking ahead, since juvenile psychosocial factors and current school pressure at follow-up were not significantly positively related, juvenile MSK pain seem to be at low risk to become chronic if psychosocial stressors do not become chronic. The change in psychosocial stressors across time and course of MSK pain should be studied in future prospective studies with use of shorter follow-ups in order to estimate the interplay of exposition and reversibility.

In line with Erne and Elfering's baseline analyses [9] we found that school pressure remained an independent risk factor for development of MSK pain five years later, even in adolescents that were recommended to higher school levels with more resources to cope with the pressure. The availability of additional information on adolescents' sleep behaviour and sleep quality meant that at follow-up we were able to demonstrate that sleep quality mediated the relationship between school pressure and MSK pain, even after controlling for age, sex and total baseline MSK pain. Although sleep disorders are relatively common during childhood, chronic sleep disorders are not and if, often linked to psychiatric disorders [21]. Sleep indicators such as short-term sleep quantity or quality have within the last decade been shown to be strongly associated with the development of MSK pain in children and adolescents [22-24]. Lack of sleep and short-term poor sleep quality have been characterised as risk factors for the development of spinal pain in children and adolescents over two years [25] and five years [26]. It is apparent that there may be a vicious circle in operation in relation to MSK pain. High school pressure may lead to a delayed sleep onset, lack of sleep or poor sleep quality and hence to a reduction in pain threshold, increased fatigue and reduced ability to concentrate - which, in turn, may increase school pressure [23]. Thus, it is likely that sleep was 
Musculoskeletal pain in adolescents and young adults 9

already acting as a mediator of the association between school pressure and MSK pain at baseline, which should be addressed next.

\section{Strengths and Limitations}

This article has several strengths, including the longitudinal approach and good five-year retention rate. We have extended the findings of Erne and Elfering [9], contributing new information to the ongoing discussion about risk factors for the development of juvenile MSK pain.

A main limitation is the small sample size, which can be explained by its longitudinal design. Almost one third of all participants were lost to follow-up due to invalid or change of address. With regard to the denial of corporation, Swiss law prioritises the protection of personal privacy rather than availability of large databases for research, even in cases where the research has considerable practical relevance, for example with respect to the personal and societal costs of MSK pain. Then, some bias could arise due to the 2.5 to 3 yrs. range in the age-span of the included participants. However, we repeated the longitudinal linear regression analysis including only 11 to 12 yrs. old pupils ( $n=86,76.8 \%)$ without any changes in results. Also, no sleep variable was collected at baseline [9] due to the different focus which lied on many specific school-related (ergonomic) factors and their potential impact on the development of low back pain in school. Furthermore, a limitation could arise in the fact of using two different modes of administration paper pencil in the baseline and an electronic self-complete questionnaire in the follow-up. However, a recent meta-analysis investigating differences in these two modes did not find any noteworthy differences and concluded that both modes could be used interchangeably for research in clinic or home settings [27]. Besides, there were no noteworthy differences detected in the quality and quantity of data when face-toface interview settings with adolescents were compared to an online administration [28]. Further research will have to confirm these findings in the healthcare sector. Finally, two important variables related to juvenile MSK were not taken into account in the calculations, physical activity and possible physical restrictions due to MSK. However, participants did not deviate from the norm for both variables in the baseline surveys and five years later. For this reason, it is not surprising that the two variables, when integrated into the analyses and statistically controlled for, had no impact on the results.

\section{Implications}


Musculoskeletal pain in adolescents and young adults 10

Even in childhood and adolescence MSK pain is a risk factor for further MSK pain episodes. Adult MSK pain prevention should start with prevention of juvenile MSK pain [3]. It may be easier to address the risks represented by high school pressure and poor sleep by offering extra tuition for pupils and providing education in sleep hygiene and the option to monitor sleep with easy-to-use sleep actigraphy functions than to improve low socioeconomic status, which is another important predictor of MSK pain in adolescents [6]. It should be noted, however, that sleep deprivation during adolescence is a Pandora's box, as it is related to excessive consumption of electronic media and to several negative health outcomes [24].

\section{Conclusion}

Juvenile MSK pain predicts subsequent MSK pain episodes, even after five years. Furthermore, school pressure is related to MSK pain and this relationship seems to be mediated by subjective sleep quality. Efforts to prevent juvenile MSK pain should take into account both school pressure and sleep quality. 


\section{References}

1. Kamper SJ, Yamato TP, Williams CM (2016) The prevalence, risk factors, prognosis and treatment for back pain in children and adolescents: An overview of systematic reviews. Best Pract Res Clin Rheumatol 30:1021-1036. https://doi.org/10.1016/j.berh.2017.04.003

2. Lazary A, Szövérfi Z, Szita J, Somhegyi A, Kümin M, Varga PP (2014) Primary prevention of disc degeneration-related symptoms. Eur Spine J 23(Suppl 3):S385-S393. https://doi.org/10.1007/s00586013-3069-X

3. Kamper SJ, Henschke N, Hestbaek L, Dunn KM, Williams CM (2016) Musculoskeletal pain in children and adolescents. Braz J Phys Ther 20:275-284. https://doi.org/10.1590/bjptrbf.2014.0149

4. Trevelyan FC, Legg SJ (2006) Back pain in school children-Where to from here? Appl Ergon 37:4554. https://doi.org/10.1016/j.apergo.2004.02.008

5. Clinch J, Eccleston C (2009) Chronic musculoskeletal pain in children: assessment and management. Rheumatol 48:466-474. https://doi.org/10.1093/rheumatology/kep001

6. Huguet A, Tougas ME, Hayden J, McGrath PJ, Stinson JN, Chambers CT (2016) Systematic review with meta-analysis of childhood and adolescent risk and prognostic factors for musculoskeletal pain. Pain 157:2640-2656. https://doi.org/10.1097/j.pain.0000000000000685

7. El-Metwally A, Mikkelsson M, Ståhl M, Macfarlane GJ, Jones GT, Pulkkinen L, Rose RJ, Kaprio J (2008) Genetic and environmental influences on non-specific low back pain in children: a twin study. Eur Spine J 17:502-508. https://doi.org/10.1007/s00586-008-0605-1

8. Balagué F, Troussier B, Salminen JJ (1999) Non-specific low back pain in children and adolescents: risk factors. Eur Spine J 8:429-438. https://doi.org/10.1007/s005860050201

9. Erne C, Elfering A (2011) Low back pain at school: unique risk deriving from unsatisfactory grade in maths and school-type recommendation. Eur Spine J 20:2126-2133. https://doi.org/10.1007/s00586011-1803-9

10. Zhang Y, Deng G, Zhang Z, Zhou Q, Gao X, Di L, Che Q, Du X, Cai Y, Han X, Zhao Q (2015) A cross sectional study between the prevalence of chronic pain and academic pressure in adolescents in China (Shanghai). BMC Musculoskel Dis 16:219. https://doi.org/10.1186/s12891-015-0625-Z

11. Zhou L, Huang YY, Chen DY, Zhang D, Luo QS, Wang Y, Wu Y (2018) [Correlation between both neck/shoulder and low back pain and daily behavioral habits among middle school students in Shenzhen]. Zhonghua Liuxingbingxue Zazhi 39:469-473. https://doi.org/:10.3760/cma.j.issn.0254$\underline{6450.2018 .04 .016}$ Chinese

12. Wiklund M, Malmgren-Olsson E-B, Öhman A, Bergström E, Fjellman-Wiklund A (2012) Subjective health complaints in older adolescents are related to perceived stress, anxiety and gender - a crosssectional school study in Northern Sweden. BMC Public Health 12:993. https://doi.org/10.1186/14712458-12-993

13. Østerås B, Sigmundsson H, Haga M (2015) Perceived stress and musculoskeletal pain are prevalent and significantly associated in adolescents: an epidemiological cross-sectional study. BMC Public Health 15:1081. https://doi.org/10.1186/s12889-015-2414-x

14. Torsheim T, Wold B (2001) School-related stress, school support, and somatic complaints. A general populations study. J Adolescent Res 16:293-303. https://doi.org/10.1177/0743558401163003

15. Murberg TA, Bru E (2004) School-related stress and psychosomatic symptoms among Norwegian adolescents. School Psychol Int 25:317-332. https://doi.org/10.1177/0143034304046904

16. Kottwitz MU, Rolli Salathé C, Buser C, Elfering A (2017) Emotion work and musculoskeletal pain in supermarket cashiers: a test of a sleep-mediation model. Scand J Work Organ Psychol 2:1-13. https://doi.org/10.16993/sjwop.25

17. Andersen T, Christensen FB, Høy KW, Helmig P, Niedermann B, Hansen ES, Bünger C (2010) The predictive value of pain drawings in lumbar spinal fusion surgery. Spine J 10:372-379. https://doi.org/10.1016/j.spinee.2010.02.002

18. Eschenbeck H, Lohaus A, Kohlmann CW (2007) Instrumente zur Erfassung von Stress und Coping im Kindesalter [Stress and coping assessment instruments in children]. In: Seiffge-Krenke I, Lohaus A (eds) Stress und Stressbewältigung im Kindes- und Jugendalter. Hogrefe, Göttingen, pp 29-46

19. Hayes AF (2017) Introduction to mediation, moderation, and conditional process analysis. A regression-based approach, 2nd edn. Guilford Press, New York.

20. Preacher KJ, Hayes AF (2008). Asymptomatic and resampling strategies for assessing and comparing indirect effects in multiple mediator models. Behav Res Methods 40:879-891. https://doi.org/10.3758/BRM.40.3.879 
21. Winsper, 2018. Sleep Disorders: Prevalence and Assessment in Childhood. In: Matson J. (ed.) Handbook of Childhood Psychopathology and Developmental Disabilities Assessment (p. 331-357). Cham: Springer Nature.

22. Harrison L, Wilson S, Munafò MR (2016) Pain-related and psychological symptoms in adolescents with musculoskeletal and sleep problems. Clin J Pain 32:246-253. https://doi.org/10.1097/AJP.0000000000000252

23. Silva AG, Sa-Couto P, Queirós A, Neto M, Rocha NP (2017) Pain, pain intensity and pain disability in high shool students are differently associated with physical activity, screening hours and sleep. BMC Musculoskel Dis 18:194. https://doi.org/10.1186/s12891-017-1557-6

24. Yabe Y, Hagiwara Y, Sekiguchi T, Momma H, Tsuchiya M, Kuroki K, Kanazawa K, Koide M, Itaya N, Itoi E, Nagatomi R (2018) Late bedtimes, short sleeping time, and longtime video-game playing are associated with low back pain in school-aged athletes. Eur Spine J 27:1112-1118. https://doi.org/10.1007/s00586-017-5177-5

25. Auvinen JP, Tammelin TH, Taimela SP, Zitting PJ, Järvelin M-R, Taanila AM, Karppinen JI (2010) Is insuffient quantity and quality of sleep a risk factor for neck, shoulder and low back pain? A longitudinal study among adolescents. Eur Spine J 19:641-649. https://doi.org/10.1007/s00586-0091215-2

26. Szita J, Boja S, Szilagyi A, Somhegyi A, Varga PP, Lazary A (2018) Risk factors of non-specific spinal pain in childhood. Eur Spine J 27:1119-1126. https://doi.org/10.1007/s00586-018-5516-1

27. Rutherford C, Costa D, Mercieca-Bebber R, Rice H, Gabb L, King M (2016) Mode of administration does not cause bias in patient-reported outcome results: a meta-analysis. Qual Life Res 25:559-574. https://doi.org/10.1007/s11136-015-1110-8

28. Shapka JD, Domene JF, Khan S, Yang LM (2016) Online versus in-person interviews with adolescents: An exploration of data equivalence. Comput Hum Behav 58:361-367. https://doi.org/10.1016/j.chb.2016.01.016 
Musculoskeletal pain in adolescents and young adults 13

Figure 1. Recruitment of participants 
Figure 2. Mediation model in predicting the total MSK pain at follow-up in $N=107$, with bold covariates indicating significant processes. Unstandardized regression coefficients (B) are estimated by use of PROCESS 2.13, Model 4 with 5000 bootstrap samples for bias corrected estimation of the confidence interval of indirect effect [19]. $\mathrm{c}=$ unstandardized regression coefficient (B) in prediction of the total MSK pain when the mediator sleep quality is not included in the model. $\mathrm{c}^{\prime}=$ unstandardized regression coefficient (B) in prediction of the total MSK pain when the mediator sleep quality is included in the model. $\mathrm{BSL}=$ Baseline, $\mathrm{FU}=$ follow-up, $\mathrm{SE}_{\mathrm{B}}=$ Standard error of estimation, $\mathrm{CI}=95 \%$ confidence interval, $\mathrm{R}^{2}=$ explained variance, ${ }^{\dagger} p<.10,{ }^{*} p<.05,{ }^{* *} p<.01,{ }^{* * *} p<.001$, two tailed. 
Musculoskeletal pain in adolescents and young adults 15

Table 1. Socio-demographic characteristics of the study samples 
Musculoskeletal pain in adolescents and young adults 16

Table 2. Intercorrelations of included variables 
Musculoskeletal pain in adolescents and young adults 17

Table 3. Stepwise linear regression analysis $(N=107)$ on outcome variable total MSK pain at follow-up 